\title{
A|P| $\begin{aligned} & \text { The Journal of } \\ & \text { Chemical Physics }\end{aligned}$
}

\section{How a short double-stranded DNA bends}

Jaeoh Shin, O-Chul Lee, and Wokyung Sung

Citation: The Journal of Chemical Physics 142, 155101 (2015); doi: 10.1063/1.4916379

View online: http://dx.doi.org/10.1063/1.4916379

View Table of Contents: http://scitation.aip.org/content/aip/journal/jcp/142/15?ver=pdfcov

Published by the AIP Publishing

\section{Articles you may be interested in}

Electrophoretic mobility of semi-flexible double-stranded DNA in defect-controlled polymer networks:

Mechanism investigation and role of structural parameters

J. Chem. Phys. 142, 234904 (2015); 10.1063/1.4922367

Characterizing the bending and flexibility induced by bulges in DNA duplexes

J. Chem. Phys. 142, 165101 (2015); 10.1063/1.4917199

Helix untwisting and bubble formation in circular DNA

J. Chem. Phys. 138, 205103 (2013); 10.1063/1.4807381

Condensation of circular DNA

J. Chem. Phys. 138, 164903 (2013); 10.1063/1.4802004

DNA nanomechanics: How proteins deform the double helix

J. Chem. Phys. 130, 135102 (2009); 10.1063/1.3082157

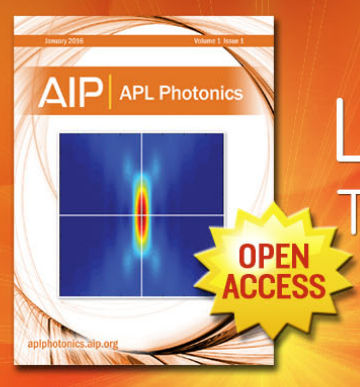




\title{
How a short double-stranded DNA bends
}

\author{
Jaeoh Shin, ${ }^{1}$ O-Chul Lee, ${ }^{1}$ and Wokyung Sung ${ }^{1,2}$ \\ ${ }^{1}$ Department of Physics and POSTECH Center for Theoretical Physics, \\ Pohang University of Science and Technology, Pohang 790-784, South Korea \\ ${ }^{2}$ IBS Center for Self-assembly and Complexity, Pohang 790-784, South Korea
}

(Received 30 October 2014; accepted 2 March 2015; published online 17 April 2015)

\begin{abstract}
A recent experiment using fluorescence microscopy showed that double-stranded DNA fragments shorter than 100 base pairs loop with the probabilities higher by the factor of $10^{2}-10^{6}$ than predicted by the worm-like chain (WLC) model [R. Vafabakhsh and T. Ha, Science 337, 1101(2012)]. Furthermore, the looping probabilities were found to be nearly independent of the loop size. The results signify a breakdown of the WLC model for DNA mechanics which works well on long length scales and calls for fundamental understanding for stressed DNA on shorter length scales. We develop an analytical, statistical mechanical model to investigate what emerges to the short DNA under a tight bending. A bending above a critical level initiates nucleation of a thermally induced bubble, which could be trapped for a long time, in contrast to the bubbles in both free and uniformly bent DNAs, which are either transient or unstable. The trapped bubble is none other than the previously hypothesized kink, which releases the bending energy more easily as the contour length decreases. It leads to tremendous enhancement of the cyclization probabilities, in a reasonable agreement with experiment. @ 2015 AIP Publishing LLC. [http://dx.doi.org/10.1063/1.4916379]
\end{abstract}

\section{INTRODUCTION}

The DNA molecule is a double helix of two singlestranded (ss) chains paired by hydrogen bondings and stacking interactions along complementary base-pairs (bp). ${ }^{1}$ Mechanically, double-stranded (ds) DNA is a stiff chain molecule having the persistence length of about $50 \mathrm{~nm}$ or $150 \mathrm{bp}$. In a living cell, it is often tightly bent on nanometer scales for biological functions; ${ }^{2-4}$ for example, in a nucleosome, a pivotal element for DNA packing in a nucleus is wrapped around proteins of about $10 \mathrm{~nm}$ size called histones; it can loop over a length of $10 \mathrm{~nm}$ mediated by transcription factors for gene regulation. ${ }^{5}$

According to the worm-like chain (WLC) model, ${ }^{6}$ in which the dsDNA is treated as an elastic rod, it is energetically improbable for the DNA to bend over the length scales much shorter than the persistence length. Cloutier and Widom ${ }^{7}$ reported the cyclization probability (called $j$-factor) of bare DNA fragments with the lengths less than $100 \mathrm{bp}$ to be many orders of magnitude higher than those predicted by the WLC model. ${ }^{8,9}$ Subsequent work by Du et al. ${ }^{10}$ however, claimed that this finding was an artifact of the high ligase concentration used to assay the $j$-factor. Nevertheless, controversy related to the $j$-factor and the associated flexibility of short DNA fragments persisted. ${ }^{11-13}$ Recently, Vafabakhsh and $\mathrm{Ha}^{14}$ vindicated the finding of Cloutier and Widom, using single molecule fluorescence resonance energy transfer (FRET).

The high flexibility of dsDNA is thought to be due to local denaturation. As widely have been studied, ${ }^{15-19}$ thermal excitation can induce global denaturation above the melting temperature of about $350 \mathrm{~K}$ as well as local opening of the duplex structure, called a thermal bubble, at the physiological temperature. Because an energy of about $10 k_{\mathrm{B}} T$ is required to initiate a bp opening, the bubbles rarely occur at physiological temperature. ${ }^{20,21}$ Bubbles form preferentially in ATrich regions where hydrogen bonding is weaker, but decay quickly, ${ }^{22}$ with little effect on DNA stability. Yan and Marko ${ }^{23}$ and Ranjith et al. ${ }^{39}$ hypothesized that a kink consisting of a ssDNA bubble can release the bending energy of highly bent ds part and can greatly enhance the cyclization probability. Similarly, Wiggins et al. ${ }^{24,25}$ introduced a generalized WLC model that predicted a higher $j$-factor and other mechanical properties. These studies, however, did not explain how the kinks spontaneously evolved from the seemingly ignorable thermal bubbles and are characterized by the involved physical parameters systematically without fitting. In particular, in these studies, the initiation energy of bubble was not considered, so that the cooperativity of bubbles was missed.

Experimentally, Du et al..$^{26}$ found structural disruptions in circular DNAs of the length shorter than $65 \mathrm{bp}$, but not in longer one. Later, MD simulation ${ }^{27}$ have shown that the defect is none other than a single kink with an open bp. Qu et al. ${ }^{28,29}$ studied the bending energy of a short dsDNA fragment with the ends linked by ss fragment so that the hybrid is considered to be in a D-shape. It was found that below a critical ssDNA length, the dsDNA develops a kink, with their nature unexplained, and the bending energy increases linearly with the bending angle instead of quadratically. A recent simulation has shown that in a short DNA fragment constrained to be a circle, a bubble tends to form more easily than in free one. ${ }^{30}$

Despite extensive studies of the elasticity of short DNA, the origin of the high flexibility and the associated kinks is not fully understood (for recent reviews, see Refs. 3 and 4). To elucidate this point along with a variety of the related phenomena in tightly bent, short DNA fragment (called as 
"short DNA" from now on), we start with an Ising model without fitting parameters. We focus on the number of unbound $\mathrm{bp}$, or the bubble size, as the primary degree of freedom and study how it is coupled to the bending mode by developing an effective Hamiltonian for a short DNA. We find that bending stress reduces the bp interaction strengths (Ising parameters) so as to enhance local denaturation of the DNA. While in a short DNA fragment uniformly bent above a critical angle, the bubble is unstable and evolves into global denaturation, in the DNA allowed to bend non-uniformly, it grows to a finite portion and is trapped for long time, which forms a flexible hinge, a kink. We assess the nature and condition of emergence of the kink, which can significantly lower the free energy of cyclization.

The outline of the paper is as follows. In Sec. II, we consider short DNA uniformly bent, either into a circle or with free ends. In Sec. III, we study the bubble behavior in non-uniformly bent DNA and the emergent kink analytically. We discuss the validity of our kink idea in view of recent experiment on short DNA cyclization. We summarize the paper in Sec. IV.

\section{MODEL FOR UNIFORMLY BENT DNA FRAGMENTS}

We start with an Ising type effective Hamiltonian for a dsDNA fragment of $N$ bases in the absence of external bending, ${ }^{31,32}$

$$
\begin{aligned}
H_{0} & =-\sum_{i=1}^{N-1}\left[J_{0} \sigma_{i} \sigma_{i+1}+\frac{K_{0}}{2}\left(\sigma_{i}+\sigma_{i+1}\right)\right]-\mu \sum_{i=1}^{N} \sigma_{i} \\
& =-\sum_{i=1}^{N-1}\left[J_{0} \sigma_{i} \sigma_{i+1}+\frac{L_{0}}{2}\left(\sigma_{i}+\sigma_{i+1}\right)\right]-\frac{\mu}{2}\left(\sigma_{1}+\sigma_{N}\right),
\end{aligned}
$$

where $\sigma_{i}=1$ for bound $\mathrm{bp}$ and $\sigma_{i}=-1$ for unbound $\mathrm{bp}$ (Fig. 1). $2 J_{0}$ accounts for the domain wall energy and $2 K_{0}$ is the difference in stacking energy between the bound bps and unbound bps, while $2 \mu$ is the bp binding energy. In Ref. 31, the local thermal undulation (orientational fluctuation) that underlies the Hamiltonian is effectively incorporated by the Ising parameters, $J_{0}, K_{0}$, and $L_{0} \equiv \mu+K_{0}$. In this work, we adopt their values $J_{0}=2.83 k_{\mathrm{B}} T, L_{0}=0.184 k_{\mathrm{B}} T$, and $\mu=1.61 k_{\mathrm{B}} T$, at $T=310 \mathrm{~K}$, obtained from thermal melting of DNA fragments consisting of AT sequences (poly(dA)poly(dT)). In the limit $N \rightarrow \infty$, the standard Ising model

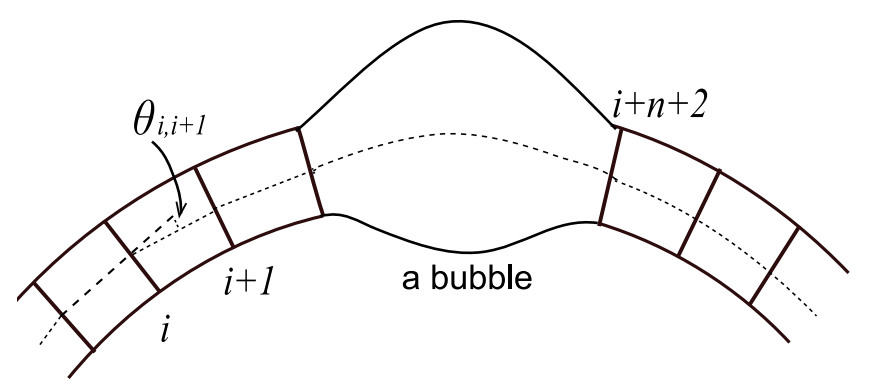

FIG. 1. A DNA fragment with a bubble of size $n$ bent by a local angle $\theta_{i, i+1}$. calculation $^{33}$ yields the fraction of bound bp as

$$
c=\sinh \left(\beta L_{0}\right) / \sqrt{\sinh ^{2}\left(\beta L_{0}\right)+\exp \left(-4 \beta J_{0}\right)},
$$

signifying the melting condition $L_{0}=0$. Incorporating the effect of entropy from the thermal undulation, we put

$$
L_{0}=\mu\left(1-T / T_{m}^{\infty}\right),
$$

where $T_{m}^{\infty} \approx 350 \mathrm{~K}$ is the melting temperature for an infinitely long DNA.

Under an applied bending stress, the net Hamiltonian is given by $H=H_{0}+H_{b}$, where

$$
H_{b}=\frac{1}{2} \sum_{i=1}^{N-1} \kappa\left(\sigma_{i}, \sigma_{i+1}\right) \theta_{i, i+1}^{2}
$$

is the bending energy. Here, $\theta_{i, i+1}$ is the bending angle of the center line (dotted line in Fig. 1) adjoining the $i$ and $i+1$ bases with respect to the foregoing segment, $\kappa\left(\sigma_{i}, \sigma_{i+1}\right)$ accounts for the associated bending rigidity: $\kappa(1,1)=\kappa_{d}$, $\kappa(-1,-1)=\kappa_{s}$, where $\kappa_{d}$ and $\kappa_{s}$ are the bending rigidities in the ds chain and twice that of ss chain, respectively. Finally, $\kappa(1,-1)=\kappa(-1,1)=\kappa_{d s}$. The bending rigidities are given by $\kappa=k_{\mathrm{B}} T L_{p} / l$, where $L_{p}$ is the persistence length and $l$ is the inter-base distance of about $0.34 \mathrm{~nm}$. Therefore, we use $\kappa_{d} / k_{\mathrm{B}} T=147$ and $\kappa_{s} / k_{\mathrm{B}} T=8.45$, corresponding to experimental values of ds and ss persistence lengths, $50 \mathrm{~nm}$ and $3 \mathrm{~nm}$, respectively, at a temperature $T=310 \mathrm{~K}$. Due to very large difference between $\kappa_{d}$ and $\kappa_{s}, \kappa_{d s}$ is well approximated by $\kappa_{s}$ (see Appendix A).

We investigate how the bending couples with emergence of open base pairs to induce changes in elastic properties. We first consider the total bending, made by an angle $\Phi$, is constrained to be uniform in a two dimensional plane, which is appropriate to the linear DNA fragment bent into a circular arc. The effect of local angle fluctuation (undulation) about the average bending angle is incorporated by the Ising parameters as in the absence of external bending. The Hamiltonian can be written as

$$
\begin{aligned}
H= & -\sum_{i=1}^{N-1}\left[J \sigma_{i} \sigma_{i+1}+\frac{L}{2}\left(\sigma_{i}+\sigma_{i+1}\right)\right] \\
& -\frac{\mu}{2}\left(\sigma_{1}+\sigma_{N}\right)+(N-1) \Gamma .
\end{aligned}
$$

Here,

$$
\begin{gathered}
J=J_{0}-\frac{1}{8} \Delta \kappa \theta^{2}, \\
L=L_{0}-\frac{1}{4} \Delta \kappa \theta^{2}, \\
\Gamma=\frac{1}{8}\left(\kappa_{d}+3 \kappa_{s}\right) \theta^{2},
\end{gathered}
$$

given in terms of uniform segmental bending angle $\theta \equiv \Phi /(N$ $-1)$, and $\Delta \kappa \equiv \kappa_{d}-\kappa_{s}$. The partition function is given by

$$
Z=\sum_{\left\{\sigma_{i}\right\}} \exp (-\beta H)
$$

where the summation is over all possible bp states $\sigma_{i}= \pm 1$ and $\beta=1 /\left(k_{\mathrm{B}} T\right)$. From this, the thermodynamic free energy $F$ and 
the fraction of open bp $f$ are obtained as

$$
F=-k_{\mathrm{B}} T \log Z
$$

and

$$
f=\frac{1}{2}(1-c),
$$

where

$$
c=\frac{1}{N}\left\langle\sum \sigma_{i}\right\rangle=-\frac{k_{\mathrm{B}} T}{N} \frac{\partial F}{\partial L} .
$$

We consider two different kinds of boundary conditions, periodic boundary $\left(\sigma_{1}=\sigma_{N}\right)$ for circular DNA and the closed ends $\left(\sigma_{1}=\sigma_{N}=1\right)$ for linear DNA. The partition function is evaluated by the transfer matrix method as sketched in Appendix B.

\section{A. Circular DNA}

First, we consider a uniformly bent circular DNA, which is relevant for the DNA of wrapped around histone protein in eukaryotic cells and also can be made artificially. ${ }^{26}$ In this case, the end effect is absent $\left(\sigma_{i}=\sigma_{N}\right)$ and under the approximation that the $N$ is sufficiently large to allow $Z \approx \lambda_{+}^{N-1}$ (see Appen$\operatorname{dix} \mathrm{B})$, the fraction of closed base pairs, $c$, is given by ${ }^{33}$

$$
c=\sinh (\beta L) / \sqrt{\sinh ^{2}(\beta L)+\exp (-4 \beta J)} .
$$

The equation implies that the melting condition $c=0$ is simply $L=0$, rewritten in view of Eqs. (2) and (5b) with $\Phi=2 \pi$,

$$
\mu\left(1-T / T_{m}^{\infty}\right)-\frac{\Delta \kappa}{4}\left(\frac{2 \pi}{N}\right)^{2}=0 .
$$

It predicts the critical chain length

$$
N_{c}=\pi\left[\Delta \kappa / \mu\left(1-T / T_{m}^{\infty}\right)\right]^{1 / 2}
$$

below which a circular DNA melts. With the parameters, we adopt $N_{c} \simeq 86$ at $T=310 \mathrm{~K}$. This value is comparable to the critical length of circular DNA in the experiment, at which disruptions of helical structure are detected for $N=64-65$ but not for $N>85-86 .{ }^{26}$ The left hand side of Eq. (11) can be written as $\mu\left(1-\frac{T}{T_{m}}\right)$, where $T_{m}$ is the melting temperature of the circular DNA of finite length, so that by putting $T=T_{m}$, it yields

$$
T_{m}=T_{m}^{\infty}\left[1-\frac{\Delta \kappa}{4 \mu}\left(\frac{2 \pi}{N}\right)^{2}\right] .
$$

It predicts the melting temperature depression induced by bending. In Fig. 2, we show thermal melting curve of circular DNA of different lengths $N$. As the chain gets shorter (higher bending), the melting temperature (marked by crosses) decreases. This finding is consistent with a recent simulation study on circular $\mathrm{DNA}^{30}$ which shows that as the chain size decreases, the bubble more easily occurs and the melting temperature decreases.

\section{B. End-bound uniformly bent DNA}

In the following, we consider the condition of closed boundary, $\sigma_{1}=\sigma_{N}=1$, which is relevant for the DNA loop formation in cell having the ends bound by proteins. For a

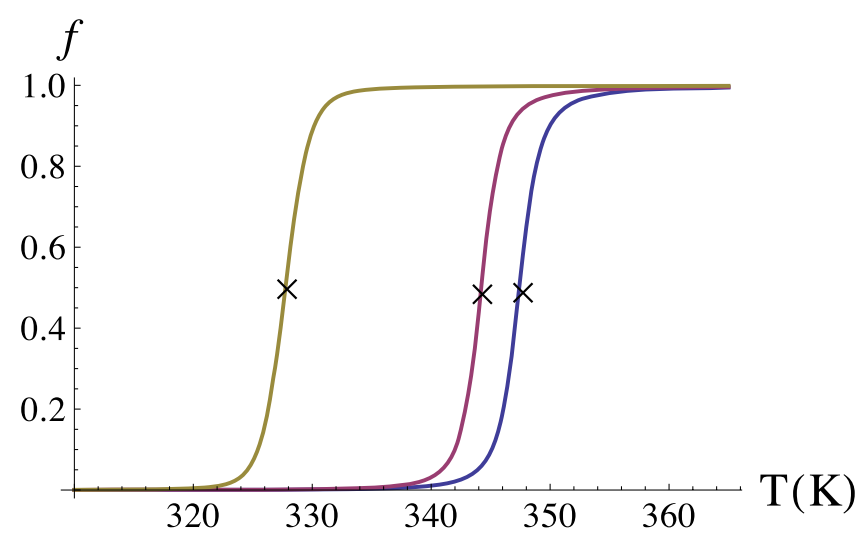

FIG. 2. The fraction of bubble $f$ as a function of temperature $T$ for circular DNA. From right to left, the chain lengths are $N=360$ (blue), $N=240$ (violet), and $N=120$ (yellow). Marked as crosses are the melting points.

linear short DNA with $N=60$ bent into a circular arc by an angle $\Phi$, we numerically obtain the free energy as a function of $\Phi$ (incorporating two eigenvalues of the transfer matrix, Appendix B), as depicted by Figure 3(a). For small bending, the free energy (solids lines) increases quadratically with $\Phi$
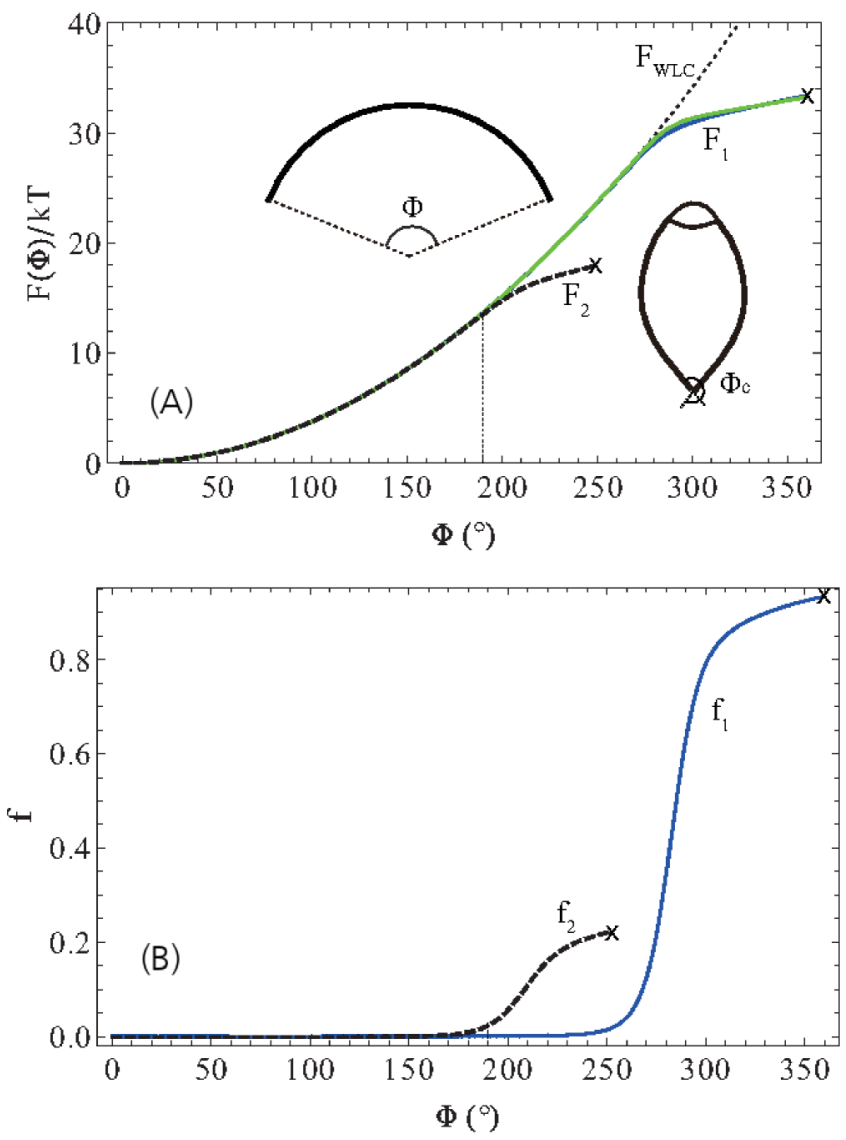

FIG. 3. (a) Free energy of uniformly bent DNA ( $F_{1}$ solid lines) and nonuniformly bent DNA ( $F_{2}$ dashed line) as a function of bending angle $\Phi$, for $N=60$. Deviation from the WLC model (dotted line) occurs at a smaller bending angle in the latter case. Insets are schematic diagrams of uniformly bent (left) and looped DNA via kink-induced non-uniform bending (right). (b) The bubble fraction $f$ or the bubble size $\langle n\rangle=N f$ for uniformly bent DNA ( $f_{1}$ solid lines) and kink-induced non-uniformly bent DNA $\left(f_{2}\right.$ dashed line). We used $T=310 \mathrm{~K}, J_{0}=2.83 k_{\mathrm{B}} T, L_{0}=0.184 k_{\mathrm{B}} T$, and the persistence lengths for ds and ss are $50 \mathrm{~nm}$ and $3 \mathrm{~nm}$, respectively. 
with virtually no deviation from the bubble-free circular WLC bending energy,

$$
F_{W L C}(\Phi)=\frac{1}{2} \kappa_{d} \frac{\Phi^{2}}{N-1}
$$

(indicated by the dotted line). As the bending increases above a critical angle $\Phi_{1} \approx 260^{\circ}$, however, it increases linearly with $\Phi$, below the WLC result. As for the unbound bp, $f$ (solid line in Fig. 3(b)) is less than $10^{-5}$ below the $\Phi_{1}$, but for bending above this critical angle, it increases abruptly to the melting point defined by $c=0$ or $f=1 / 2$ and global denaturation $f=1$, reminiscent of sharp melting transition. ${ }^{15}$ It evidently demonstrates how the uniform bending triggers ambient, but seemingly invisible open bp to evolve into denaturation bubbles, thereby release the bending energy.

More direct, analytical modeling for the bending energy release mechanism is given below in terms of the nature of the bubble that emerges. An energy $4 J_{0}$ is required to initiate a bp opening, whereas the bp pairing energy is $2 L_{0}$. Because the former is much larger than the latter at $T=310 \mathrm{~K}$, an open $\mathrm{bp}$, once formed, tends to grow rather than multiple open $\mathrm{bp}$ emerge separately. For short DNAs, therefore, it is reasonable that the open bps can exists only within a domain, called as a bubble, as also shown in Refs. 30 and 34. The size of bubble, $n$, defined by the number of the open bp, is a new degree of freedom that replaces $\sigma_{i}$ 's in the Ising Hamiltonian. The Hamiltonian, with $\sigma_{i}=1$ assigned to the ds part and $\sigma_{i}=-1$ to the bubble domain of size $n$, becomes, apart from an irrelevant constant term,

$$
\begin{aligned}
\mathcal{F}_{1}(n ; \Phi) & =F_{W L C}(\Phi), & & n=0 \\
& =\mathcal{F}_{B}(n)+\mathcal{F}_{b}(n ; \Phi), & & n \geq 1 .
\end{aligned}
$$

Here,

$$
\mathcal{F}_{b}=\frac{1}{2} \kappa_{d}\left(\frac{\Phi}{N-1}\right)^{2}(N-n-2)+\frac{1}{2} \kappa_{s}\left(\frac{\Phi}{N-1}\right)^{2}(n+1)
$$

is identified precisely as the bending energy and

$$
\mathcal{F}_{B}=4 J_{0}+2 L_{0} n
$$

is the free energy of bubble formation. Then, we obtain the partition function

$$
Z_{1}=\sum_{n=0}^{N-2} e^{-\beta \mathcal{F}_{1}(n ; \Phi)}
$$

and, there from the net free energy,

$$
\begin{aligned}
F_{1}(\Phi) & =-k_{\mathrm{B}} T \ln \left[\sum_{n=0}^{N-2} e^{-\beta \mathcal{F}_{1}(n ; \Phi)}\right] \\
& =F_{W L C}(\Phi)-k_{\mathrm{B}} T \ln \left[1+\sum_{n=1}^{N-2} e^{-\beta \Delta \mathcal{F}_{1}(n ; \Phi)}\right]
\end{aligned}
$$

where

$$
\Delta \mathcal{F}_{1}(n ; \Phi)=\mathcal{F}_{1}(n ; \Phi)-F_{W L C}(\Phi) .
$$

From Eq. (16), we rewrite the above as

$$
\Delta \mathcal{F}_{1}(n ; \Phi)=4 J+2 L n,
$$

where

$$
\begin{aligned}
& 4 J=4 J_{0}-\frac{\Delta \kappa}{2}\left(\frac{\Phi}{N-1}\right)^{2}, \\
& 2 L=2 L_{0}-\frac{\Delta \kappa}{2}\left(\frac{\Phi}{N-1}\right)^{2}
\end{aligned}
$$

are none other than the effective bubble initiation energy and bp unbinding energy which we already encountered in Eq. (5). Indeed Eq. (19b), which can be evaluated exactly as

$$
\begin{aligned}
F_{1}(\Phi)= & F_{W L C}(\Phi) \\
& -k_{\mathrm{B}} T \ln \left[1+e^{-4 \beta J} \cdot \frac{e^{-2 \beta L}-e^{-2 \beta L(N-1)}}{1-e^{-2 \beta L}}\right],
\end{aligned}
$$

numerically overlaps with and indistinguishable from the free energy calculated from the Ising model $\left(F_{1}(\Phi) \approx F(\Phi)\right)$ as shown in Figure 3(a), validating the single bubble approximation for this short fragment. The critical angle for the deviation of $F_{1}(\Phi)$ from $F_{W L C}(\Phi)$ is now identified as $\Phi_{1} \simeq \Phi_{m}$, where

$$
\Phi_{m}=(N-1)\left(\frac{4 L_{0}}{\Delta \kappa}\right)^{1 / 2}
$$

is the angle at which the melting occurs, $L=0$. For an angle $\Phi$ smaller than $\Phi_{m}, \Delta \mathcal{F}_{1}(n)$ increases linearly with $n$ and thus has a local minimum at $n=0$, meaning that a bubble, although can be initiated and grow due to thermal fluctuation, decays very quickly. In contrast, for $\Phi>\Phi_{m}$, the free energy landscape $\Delta \mathcal{F}_{1}(n)$ is downhill, indicating that the bubble is unstable with respect to growth toward a global denaturation, consistent with the solid line in Figure 3(b). Also, the bubble growth is responsible for the negative deviation of the net free energy $F(\phi)$ from $F_{W L C}(\phi)$ as indicated in Fig. 3(a).

\section{NON-UNIFORMLY BENT SHORT DNA FRAGMENTS}

Although the uniform bending assumption is valid for certain situations such as DNA wound around histones and DNA collapsed into a toroid, ${ }^{35}$ it is not generally true for linear fragment under high bending conditions. To incorporate non-uniform bending in strong coupling with the bubble in a simplest way, we assume that the DNA is bent within the ss and ds regions, respectively, with different segmental bending angles, $\theta_{s}$ and $\theta_{d}$. This means that two ds parts between the bubble and ends are uniformly bent, which is a quite reasonable approximation for short DNA fragments we consider here. With the backbone bending energy replaced by

$$
\mathcal{F}_{b}=\frac{1}{2} \kappa_{s} \theta_{s}^{2}(n+1)+\frac{1}{2} \kappa_{d} \theta_{d}^{2}(N-n-2),
$$

the effective Hamiltonian is written as

$$
\begin{aligned}
\mathcal{F}_{2}(n, \Theta ; \Phi) & =\mathcal{F}_{W L C}(\Phi), \quad n=0 \\
& =\mathcal{F}_{B}(n)+\mathcal{F}_{b}(n, \Theta ; \Phi) \\
& =4 J_{0}+2 L_{0} n+A \Theta^{2}-2 B \Theta \Phi+C \Phi^{2}, \quad n \geq 1
\end{aligned}
$$

in terms of an order parameter

$$
\Theta \equiv\left(\theta_{s}-\theta_{d}\right)(n+1)
$$


characteristic of non-uniform bending emergence, and the total bending angle

$$
\Phi=(n+1) \theta_{s}+(N-n-2) \theta_{d} .
$$

Here,

$$
\begin{gathered}
A=\frac{N-n-2}{2(N-1)^{2}}\left(\kappa_{d}+\frac{N-n-2}{n+1} \kappa_{s}\right), \\
B=\frac{N-n-2}{2(N-1)^{2}} \Delta \kappa,
\end{gathered}
$$

and

$$
C=\frac{N-n-2}{2(N-1)^{2}}\left(\kappa_{d}+\frac{n+1}{N-n-2} \kappa_{s}\right) .
$$

The partition function and net free energy are given by

$$
\begin{aligned}
Z_{2}(\Phi) & =\sum_{n=1}^{N-2} \frac{1}{\Phi} \int_{0}^{\Phi} d \Theta e^{-\beta \mathcal{F}_{2}(n, \Theta ; \Phi)} \\
F_{2}(\Phi) & =-k_{B} T \ln \left[Z_{2}(\Phi)\right] \\
& =F_{W L C}(\Phi)-k_{B} T \ln \left[1+\sum_{n=1}^{N-2} e^{-\beta \Delta \mathcal{F}_{2}(n ; \Phi)}\right],
\end{aligned}
$$

where

$$
\begin{aligned}
\Delta \mathcal{F}_{2}(n, \Phi)= & -k_{B} T \ln \left[\frac{1}{\Phi} \int_{0}^{\Phi} d \Theta e^{-\beta \mathcal{F}_{2}(n, \Theta ; \Phi)}\right] \\
& -F_{W L C}(\Phi) .
\end{aligned}
$$

The net free energy $F_{2}(\Phi)$ for $N=60$ as a function of $\Phi$ is depicted by the dashed line in Fig. 3(a). It is virtually identical to the result from the WLC model for angles below a critical value $\Phi_{2}$; above this value, it grows linearly with $\Phi$, similar to uniformly bent case. This behavior is consistent with an observation for dsDNA bent into the D form that a "kink" developed above a critical regime. ${ }^{28,29}$ Above $\Phi_{2} \simeq 190^{\circ}$, which is smaller than $\Phi_{1}, F_{2}(\Phi)$ is much lower than $F_{1}(\Phi)$, indicating that the non-uniform bending is the equilibrium conformation. In association with this, the fraction of bubbles $f_{2}=\frac{\langle n\rangle}{N}$ is shown as a function of $\Phi$ by the dashed line in Fig. 3(b), where

$$
\langle n\rangle=\sum_{n=1}^{N-2} n \cdot e^{-\beta \Delta \mathcal{F}_{2}(n ; \Phi)} /\left(1+\sum_{n=1}^{N-2} e^{-\beta \Delta \mathcal{F}_{2}(n)}\right)
$$

is the average bubble size. $f_{2}$ appears to be close to zero for $\Phi$ below $\Phi_{2}$ and increases gradually with higher bending. This indicates that the reduction of $F_{2}(\Phi)$ below $F_{W L C}$ is due to the emergence of a bubble, similar to the case of uniformly bent DNA. However, there is a dramatic difference; the open bp here nucleates into a bubble at a smaller bending angle $\Phi_{2}$, but it undergoes a gradual increase in contrast with the sharp increase for uniform bending case, culminating in much smaller bubble size and no global melting $(f \simeq 0.2)$.

\section{A. Emergence of a kink}

In order to explore this remarkable nature of the bubble, what we can call as the kink, we study Eq. (31), the effective free energy of bubble formation. ${ }^{36}$ Figure 4 depicts $\Delta \mathcal{F}_{2}(n ; \Phi)$

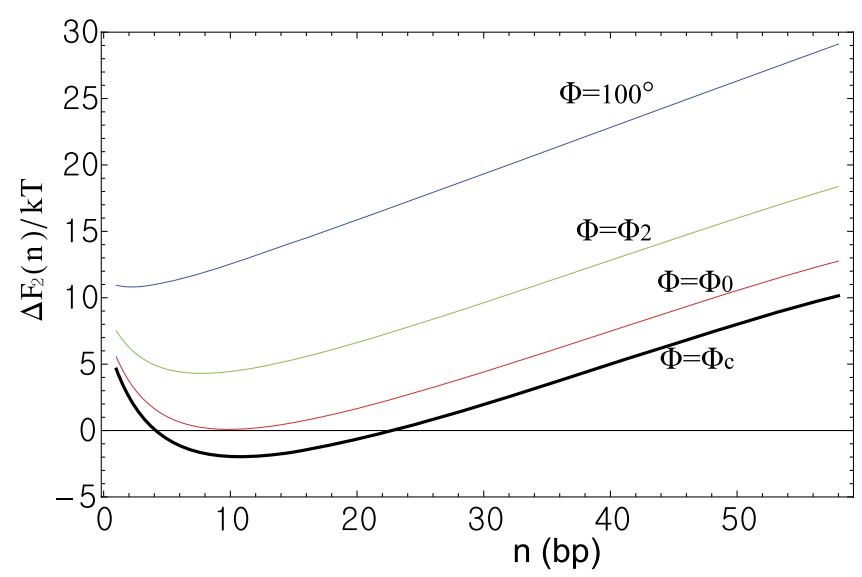

FIG. 4. Stability of a kink based on $\Delta \mathcal{F}_{2}(n)$, the free energy required to form a bubble of size $n$ in $N=60$ bp non-uniformly bent DNA. From top to bottom, bending angles are $\Phi=100^{\circ}, \Phi=\Phi_{2}\left(=200^{\circ}\right), \Phi=\Phi_{0}\left(=236^{\circ}\right)$, where $\Delta \mathcal{F}_{2}(n)$ has the minimum zero, and $\Phi=\Phi_{c}\left(=251^{\circ}\right)$ by which a loop closure forms. We used the same physical parameters as in Figure 3.

for different bending angles $\Phi$. For small $\Phi, \Delta \mathcal{F}_{2}(n, \Phi)$ increases monotonically with $n$, similar to uniform bending. Although a bubble can form by surmounting the initiation energy barrier by thermal fluctuation, it will be transient. However, as $\Phi$ increases, $\Delta \mathcal{F}_{2}(n, \Phi)$ develops a local minimum at a non-zero bubble size $m$, which is about $\langle n\rangle$. In contrast to the bubble formed in the case of uniformly bending, once a bubble nucleates to this size, it can have a long life time because there is an energy barrier to shrinkage $(n \rightarrow 0)$. As the bending angle increases further, the minimum becomes negative, meaning that the bubble with a finite size $m$ is trapped for a long time and does not lead to global denaturation unlike that in uniform bending.

To understand analytically the role the kink plays, we note that the integral in Eq. (31) is dominated by the contribution of $\Theta=\Theta_{m}$, where $\mathcal{F}_{2}(n, \Theta ; \Phi)$ is the minimum. This leads to

$$
\Delta \mathcal{F}_{2}(n ; \Phi) \approx \Delta \mathcal{F}_{b}(n ; \Phi)+\mathcal{F}_{B}(n) .
$$

Here,

$$
-\Delta \mathcal{F}_{b}=\frac{\Phi^{2}}{2(N-1)}\left[(n+1) \Delta \kappa+\frac{(N-n-2)(\Delta \kappa)^{2}}{\Delta \kappa+\kappa_{s}\left(\frac{N-1}{n+1}\right)}\right]
$$

is the bending energy release facilitated by the kink, which is enhanced for short DNA beyond that of uniform bending (the first term in [..]). The critical angle $\Phi_{0}$, above which $\Delta \mathcal{F}_{2}$ is negative and the kink becomes stable, decreases as $N$ decreases. We rewrite the effective energy of a kink formation as

$$
\Delta \mathcal{F}_{2}(n ; \Phi)=4 J+2 L n
$$

The effective bubble initiation energy is given by

$$
4 J=4 J_{0}-\frac{\Delta \kappa}{2} \frac{\Phi^{2}}{N-1}\left[1+\frac{\Delta \kappa(N-2)}{\Delta \kappa+(N-1) \kappa_{s}}\right]
$$

which, for a short DNA, tends to be increasingly lower than $4 J_{0}$ for free DNA as well as

$$
4 J_{0}-\frac{\Delta \kappa}{2}\left(\frac{\Phi}{N-1}\right)^{2}
$$


for the uniformly bent case. These results explain how the kink in shorter DNA more easily forms and stabilizes at lower bending, by releasing more readily the bending energy. Because $\theta_{s}=\left(\kappa_{d} / \kappa_{s}\right) \theta_{d}$ for the free energy minimum, the bending curvature is localized preferably on the kink rather than distributed over the remaining ds region. In the right inset of the Figure 3(a), we show the looped DNA conformations calculated for the kink located in the middle. Because of the high bending localized on the kink, a closure forms at the angle $\Phi_{c} \approx 251^{\circ}$ less than $360^{\circ}$, which additionally reduce cyclization free energy to much less than that of uniformly bent DNA.

\section{B. Relevance to cyclization experiment}

Finally, we discuss validity of the kink idea in view of the recent FRET experiment on the cyclization of short DNA with $N=67-106 \mathrm{bp} .{ }^{14}$ In the experiment, the cyclization probability ( $j$-factor) showed a weak dependence on the chain length $N$ and had magnitude $10^{2}-10^{6}$ times higher than the WLC model predictions. For a fixed $N$, it has shown that depending on the bp sequence $j$-factor can vary over as large as two orders of magnitude. The DNA ends have complementary single-strand overhangs. Because of that the DNA can make a loop once two ends are close enough, not necessarily aligned parallel as in the "tear-drop" shape in the WLC model. ${ }^{23}$

Theoretically, the $j$-factor is given by $j=\frac{Z_{C}}{Z_{0}}, 8,9$ where $Z_{c}$ and $Z_{0}$ are the partition functions of the chain with the ends closed and free, respectively. ${ }^{37}$ For short chain, $Z_{c}$ is approximated by the chain with a single kink, so that the cyclization probability can be approximated as $j \approx \exp \left(-\beta F_{2}\left(\Phi_{c}\right)\right)$. Here, $F_{2}\left(\Phi_{c}\right)$ is the free energy of bending with a kink we evaluated above for the angle of closure $\Phi_{c}$ which is less than $360^{\circ}$ because of the non-uniformity of the bending. Though the looping probability is strongly dependent on the position of the bubble, ${ }^{39}$ for simplicity, we assumed that a kink is located in the center of the fragment and numerically obtained the $F_{2}$ from Eq. (30b) for a number of different $N$ using the aforementioned physical parameter values. The $j$-factor obtained is depicted by the solid curve in Fig. 5 and compared with the experimental result (squares).

As the chain length becomes shorter than $N=100 \mathrm{bps}$, $j$-factor appears to be quite insensitive to the chain length in a way similar to the experiment ${ }^{14}$ but increased slightly as it is

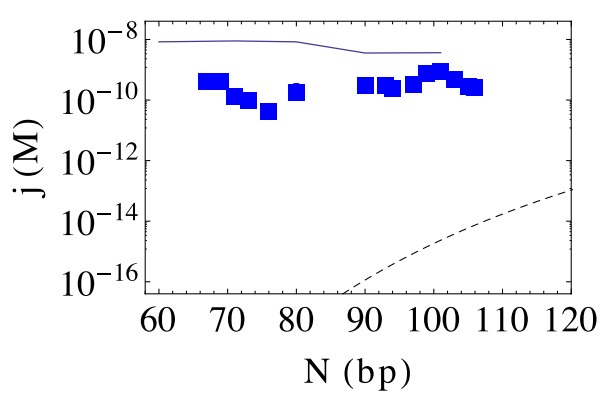

FIG. 5. The cyclization probability $j$ vs. DNA length from our model (solid line) compared with experimental data of Ref. 14 shown by squares. We used the same physical parameter as before. The dashed line is the WLC model prediction. $^{8}$ shortened from $110 \mathrm{bp}$ to $60 \mathrm{bp}$. It is because $F_{2}$ consists of two parts; the WLC bending energy of duplex that decreases with the chain length $N$ and the part involving the kink formation energy $\Delta \mathcal{F}_{2}$ (see Eqs. (30b) and (33)). The kink formation energy has a negative contribution that increases as $N$ decrease due to the bending energy release (Eq. (34)), which dominates the WLC contribution in the $N$ dependence of the $j$-factor.

Also, our results are about one order of magnitude higher than the experimental data. A reason for the discrepancy can be due to the fact that, while we used the bp interaction parameters, $J_{0}$ and $L_{0}$, extracted from the homogeneous AT bps which are smaller than those of GC bp, the experiments are done with the DNA fragment of about 50\% AT content. Indeed, in a controlled experiment with the DNA fragment with homogeneous AT bps, ${ }^{14}$ the looping is faster, which results in an increase of the $j$-factor, about 35 times compared to that of the standard sample. On the other hand, since the bubble nucleates preferably in an AT rich region in a real heterogeneous DNA, the assumption of a single kink emergence precisely at the middle may be too simple.

Considering various complex factors in the experiment, including the sequence dependent bubble opening probabilities, ${ }^{7,9,19,38}$ and the relative simplicity of our theoretical model, which do not include adjustable parameters, the fair agreement is remarkable, attesting the emergent role of the single kink in cyclization.

\section{SUMMARY}

Double-stranded DNA is a fairly stiff molecule with a persistence length of about $50 \mathrm{~nm}$. Recent studies, however, have surprisingly shown that it readily bends and loops over shorter lengths for various biological functions. To elucidate the mechanism of emerging high flexibility in such short DNA fragments, we studied theoretical models. For uniformly bent cases, an Ising-like model showed that as the bending increases, the fraction of broken base pairs (bubble size) increases and above a critical bending the DNA undergoes a sharp denaturation transition far below the melting temperature of the unbent DNA. In a circular DNA, which is a typical uniformly bent case, denatures if its contour length is less than a critical value.

We also developed a single-bubble DNA model equivalent to the Ising-like model to study the interplay between the bubble and non-uniform bending. The bending with an angle lower than a critical value does not initiate the bubble and remains to be uniform, but above the angle, the bending could easily nucleate a bubble, where a relatively sharp bending curvature is induced. As the bending increases further, the bubble gradually grows to a finite size but trapped, unlike the bubbles in uniformly bent DNA, which are either transient or unstable to grow into global denaturation.

In short, we demonstrated how thermally induced open base pairs in dsDNA, despite their rare and transient existence, couple with an applied bending to spontaneously nucleate to a kink. The kink is neither a transient thermal bubble nor a permanent bend; it is a quasi-stable dynamic structure that selforganizes to reduce the enormous bending energy barrier for looping. This idea is consistent with the recent experiment on 
DNA cyclization and is further testable in quantitative details for the predesigned sequences.

\section{ACKNOWLEDGMENTS}

This work was supported by the Brain Korea 21 program and NRF.

\section{APPENDIX A: BENDING STIFFNESS AT A JOINT}

Let us consider the bending stiffness of a joint at the boundary of open and closed base pairs. In continuum limit, the correlation of tangential vector of the chain is $\langle\vec{t}(0) \vec{t}(s)\rangle$ $=e^{-s / L_{p}}$, where $L_{p}$ is a persistence length $L_{p}=\kappa l /\left(k_{\mathrm{B}} T\right), \kappa$ is bending stiffness, and $l$ is the segmental length. The center line tangential correlation between two segments composed of a ds and a ss bp segments is $\langle\vec{t}(0) \vec{t}(2 l)\rangle \equiv e^{-\frac{2 l}{L p_{e f f}}}=e^{-\frac{l}{L p_{s}}} e^{-\frac{l}{L p_{d}}}$. The effective persistence length is $L p_{\text {eff }}=2\left(\frac{L p_{s} L p_{d}}{L p_{s}+L p_{d}}\right) \simeq 2 L p_{s}$. Therefore, the bending stiffness can be approximated as $\kappa_{d s} \simeq \kappa_{s}$.

\section{APPENDIX B: TRANSFER MATRIX METHOD FOR ISING-TYPE MODEL}

The partition function of the Ising model Hamiltonian (Eq. (4)) can be written as

$$
Z=\sum_{\sigma_{i}= \pm}\left\langle V \mid \sigma_{i}\right\rangle\left\langle\sigma_{i}\left|P_{I}\right| \sigma_{2}\right\rangle \cdots\left\langle\sigma_{N-1}\left|P_{I}\right| \sigma_{N}\right\rangle\left\langle\sigma_{N} \mid V\right\rangle
$$

where

$$
P_{I}=e^{-\beta \Gamma}\left(\begin{array}{cc}
e^{\beta L+\beta J} & e^{-\beta J} \\
e^{-\beta J} & e^{-\beta L+\beta J}
\end{array}\right)
$$

is the transfer matrix of the Hamiltonian, and the summation is over all the possible bp states, $\sigma_{i}=1$ or -1 , and $|V\rangle$ is the end state vector. For closed-end, $|V\rangle=|+1\rangle=(1,0)$ and for free-end boundary, $|V\rangle=\left(e^{\mu / 2}, e^{-\mu / 2}\right)$. The transfer matrix can be expanded in terms of the eigenvectors of the transfer matrix

$$
P_{I}=\sum_{\tau= \pm} \lambda_{\tau}|\tau\rangle\langle\tau|
$$

where the eigenvalues are $\lambda_{ \pm}=e^{\beta J-\beta \Gamma}\left[\cosh (\beta L) \pm\left(\sinh ^{2}(\beta L)\right.\right.$ $\left.\left.+e^{-4 \beta J}\right)^{1 / 2}\right]$ and the eigenvectors are

$$
|+\rangle=\frac{1}{\sqrt{2 \gamma} e^{\beta J}}\left(a_{0}|U\rangle+a_{0}^{-1}|B\rangle\right)
$$

and

$$
|-\rangle=\frac{1}{\sqrt{2 \gamma} e^{\beta J}}\left(a_{0}^{-1}|U\rangle-a_{0}|B\rangle\right) .
$$

Here, $\quad \gamma=\left(\sinh (\beta L)^{2}+e^{-4 \beta J}\right)^{1 / 2}$ and $a_{0}=e^{\beta J}(\sinh (\beta L)$ $+\gamma)^{1 / 2}$ and $|U\rangle=|+1\rangle$ and $|B\rangle=|-1\rangle$ are closed and open state of base pair, respectively. Then, the partition function is

$$
Z=\left\langle V\left|P_{I}^{N-1}\right| V\right\rangle=\sum_{\tau= \pm} \lambda_{\tau}^{N-1}\langle V \mid \tau\rangle^{2}
$$

For example, with periodic boundary $\left(\sigma_{1}=\sigma_{N}\right), Z=\lambda_{+}^{N-1}$ $+\lambda_{-}^{N-1}$.

${ }^{1}$ B. Albert, A. Johnson, J. Lewis, M. Raff, K. Roberts, and P. Walter, Molecular Biology of the Cell, 4th ed. (Garland Science, New York, 2002).

${ }^{2}$ H. G. Garcia, P. Grayson, L. Han, M. Inamdar, J. Kondev, P. C. Nelson, R. Phillips, J. Widom, and P. A. Wiggins, Biopolymers 85, 115 (2006).

${ }^{3}$ J. P. Peter and L. J. Maher III, Q. Rev. Biophys 43, 23 (2010).

${ }^{4}$ A. Vologodskii and M. D. Frank-Kamenetskii, Nucleic Acid Res. 41, 6785 (2013).

5J.-F. Allmand, S. Cocco, N. Douarche, and G. Lia, Eur. Phys. J. E. 19, 293 (2006).

${ }^{6}$ O. Kratky and G. Porod, Rec. Trav. Chim. Pays-Bas. 68, 1106 (1949).

${ }^{7}$ T. E. Cloutier and J. Widom, J. Mol. Cell 14, 355 (2004).

${ }^{8}$ J. Shimada and H. Yamakawa, Macromolecules 17, 689 (1984).

${ }^{9}$ Y. Zhang and D. M. Crothers, Biophys. J. 84, 136 (2003).

${ }^{10}$ Q. Du, C. Smith, N. Shiffeldrim, M. Vologodskaia, and A. Vologodskii, Proc. Natl. Acad. Sci. U. S. A. 102, 5397 (2005).

${ }^{11}$ P. A. Wiggins et al., Nat. Nanotechnol. 1, 137 (2006).

${ }^{12}$ C. Yuan, H. Chen, X. W. Lou, and L. A. Archer, Phys. Rev. Lett. 100, 018102 (2008).

${ }^{13}$ A. J. Mastroianni, D. A. Sivak, P. L. Geissler, and A. P. Alvisatos, Biophys, J. 97, 1408 (2009).

${ }^{14}$ R. Vafabakhsh and T. Ha, Science 337, 1101 (2012).

${ }^{15}$ R. M. Wartell and A. S. Benight, Phys. Rep. 126, 67 (1985).

${ }^{16}$ M. D. Frank-Kamenetskii, Phys. Rep. 288, 13 (1997).

${ }^{17}$ T. Dauxois and M. Peyrard, Phys. Rev. E 47, R44 (1993).

${ }^{18}$ M. Peyrard, Nonlinearity 17, R1 (2004).

${ }^{19}$ S. Talukder, P. Chaudhry, R. Metzler, and S. K. Banik, J. Chem. Phys. 135, 165103 (2011).

${ }^{20}$ J. SantaLucia, Jr., Proc. Natl. Acad. Sci. U. S. A. 95, 1460 (1998).

${ }^{21}$ A. Krueger, E. Protozanova, and M. D. Frank-Kamenetskii, Biophys. J. 90, 3091 (2006).

${ }^{22}$ G. Altan-Bonnet, A. Libchaber, and O. Krichevsky, Phys. Rev. Lett. 90, 138101 (2003).

${ }^{23}$ J. Yan and J. F. Marko, Phys. Rev. Lett. 93, 108108 (2004).

${ }^{24}$ P. A. Wiggins, R. Phillips, and P. C. Nelson, Phys. Rev. E 71, 021909 (2005).

${ }^{25}$ P. A. Wiggins and P. C. Nelson, Phys. Rev. E 73, 031906 (2006).

${ }^{26}$ Q. Du, A. Kotlyar, and A. Vologodskii, Nucleic Acids Res. 36, 1120 (2008).

${ }^{27}$ J. S. Mitchell1, C. A. Laughton, and S. A. Harris, Nucleic Acids Res. 39, 3928 (2011).

${ }^{28}$ H. Qu, Y. Wang, C.-Y. Tseng, and G. Zocchi, Phys. Rev. X 1, 021008 (2011).

${ }^{29} \mathrm{H}$. Qu and G. Zocchi, EPL 94, 18003 (2011).

${ }^{30}$ O.-C. Lee and W. Sung, Phys. Rev. E 85, 021902 (2012).

${ }^{31}$ J. Palmeri, M. Manghi, and N. Destainville, Phys. Rev. Lett. 99, 088103 (2007); Phys. Rev. E 77, 011913 (2008).

${ }^{32}$ N. Destainville, M. Manghi, and J. Palmeri, Biophys. J. 96, 4464 (2008).

${ }^{33}$ R. K. Pathria, Statistical Mechanics, 2nd ed. (Elsevier, Oxford, 1996).

${ }^{34}$ O.-C. Lee, J.-H. Jeon, and W. Sung, Phys. Rev. E 81, 021906 (2010).

${ }^{35}$ B. van den Broek, M. C. Noom, J. van Mameren, C. Battle, F. C. MacKintosh, and G. J. L. Wuite, Biophys. J. 98, 1902 (2010).

${ }^{36}$ A. Hanke and R. Metzler, J. Phys. A:Math. Gen. 36, L473 (2003).

${ }^{37}$ Note that since our model DNA does not have helical structure and the two ends are not oriented parallel, the prefactor is different from those of other models. ${ }^{9,23}$

${ }^{38}$ A. Montrichok, G. Gruner, and G. Zocchi, Europhys. Lett. 62, 452 (2003).

${ }^{39}$ P. Ranjith, P. B. Sunil Kumar, and G. I. Menon, Phys. Rev. Lett. 94, 138102 (2005). 